\title{
Cutaneous angiosarcoma: report of three different and typical cases admitted in a unique dermatology clinic ${ }^{*}$
}

\author{
Aline Neves Freitas Cabral ${ }^{1}$ \\ Ana Cristina Vervloet do Amaral ${ }^{1}$ \\ Paulo Sérgio Emerich Nogueira ${ }^{1}$
}

\author{
Rafael Henrique Rocha ${ }^{1}$ \\ Karina Bittencourt Medeiros ${ }^{2}$ \\ Lucia Martins Diniz ${ }^{3}$
}

DOI: http:/ /dx.doi.org/10.1590/abd1806-4841.20175326

\begin{abstract}
Angiosarcoma is a rare and aggressive tumor with high rates of metastasis and relapse. It shows a particular predilection for the skin and superficial soft tissues. We report three distinct and typical cases of angiosarcoma that were diagnosed in a single dermatology clinic over the course of less than a year: i) Angiosarcoma in lower limb affected by chronic lymphedema, featuring Stewart-Treves syndrome; ii) a case of the most common type of angiosarcoma loated in the scalp and face of elderly man and; iii) a skin Angiosarcoma in previously irradiated breast. All lesions presented characteristic histopathological findings: irregular vascular proliferation that dissects the collagen bundles with atypical endothelial nuclei projection toward the lumen.
\end{abstract}

Keywords: Hemangiosarcoma; Lymphangiosarcoma; Lymphedema; Non-Filarial Lymphedema; Sarcoma

\section{INTRODUCTION}

Angiosarcoma (AS) is a rare and aggressive neoplasm, that originates from endothelial cells of lymphatic and blood vessels. It accounts for $5 \%$ of malignant skin tumors and less than $1 \%$ of all sarcomas. It is notable for having a predilection for the skin and superficial soft tissues. The most common sites, in decreasing order of frequency, are the skin, breast, deep soft tissues, viscera and bones. ${ }^{1-4}$

Cutaneous AS exhibits clinical heterogeneity, and affects male and female similarly at any age. However, it is most frequently located on the head ad neck region of white elderly men. ${ }^{1-3}$

Interestingly, we report three distinct and typical AS cases associated with classically described risk factors, all from a single dermatology service within less than a year.

\section{CASE REPORT}

\section{Case 1}

A A 44-year-old woman with chronic lymphedema in her left lower limb for nearly 20 years caused by Cockett's syndrome, an anatomical variation in wich the left iliac artery compresses the left iliac vein, was admitted to the dermatology service due to the development, over the preceeding four months, of a painful red-topurple macule on the affected limb. It rapidly progressed to purple extensive macules, papules and nodules lesions, some of them with a tumoral aspect. (Figure 1).

Incisional biopsies of the lesions were performed. Histopathological exams revealed, in the purple macule lesion, proliferation of irregular vascular clefts, interposed by the dermal collagen fibers, formed by endothelium with atypical nuclei, prominent toward the lumen; The tumoral lesion exhibited cohesive epithelioid masses of atypical, large, rounded cells with acidophilic cytoplasm and frequent mitotic figures. (Figure 2). Immunohistochemical analysis was negative for HHV-8 Lana-1 oncoprotein and revealed expression of CD31 and CD34.

Based on these results, it was diagnosed as epitheliod AS and, therefore, Stewart-Treves syndrome, due to the association with chronic lymphedema.

Case 2

An 80-year-old of black color man, with a poorly defined disease onset - three to eight months before admission - reported an initial reddish purple lesion on the scalp that developed into extensive, infiltrative, confluent purple-black lesions spreading from the scalp to the lower third of the face, forming an easily bleeding left periorbital mass (Figure 3, with consent).

Anatomopathological study of the skin biopsy demonstrated proliferation of irregular vascular clefts, consisting of atypical endothelium, with hyperchromatic nuclei projecting into the lumen, leading to the diagnosis of AS (Figure 4).

Case 3

A 57-year-old woman developed, over the previous three years, papulotumoral, red-to-purple, hardened, asymptomatic lesions, located on the lower lateral quadrant of the left breast. (Figure 5).

She had a personal history of infiltrating ductal carcinoma in the affected breast, diagnosed in 2008, and had undergone six cy-

\section{Received on 03.11.2015}

Approved by the Advisory Board and accepted for publication on 22.01.2016

Study conducted at Hospital Universitário Antônio Cassiano de Moraes, da Universidade Federal do Espírito Santo (Hucam-Ufes) - Vitória (ES), Brazil.

Financial support: none.

Conflict of interest: none.

Hospital Universitário Cassiano Antônio Moraes, da Universidade Federal do Espírito Santo (Hucam-Ufes) - Vitória (ES), Brazil

Professional Master's Programa in Medicine of the Universidade Federal do Espírito Santo (Ufes) - Vitória (ES), Brazil.

Universidade Federal do Espírito Santo (Ufes) - Vitória (ES), Brazil. 
cles of neoadjuvant chemotherapy, with complete clinical, radiological and pathological responses. In 2009, conservative surgery of the left breast (superior quadrantectomies) was performed with axillary emptying, followed by 30 sessions of radiotherapy. Histopathological examination of the surgical specimen showed no residual neoplasia with negative lymph nodes.

Histopathological examination of a breast papule demosntrated anastomotic suture of sinusoidal vessels, with an infiltrative aspect, coated by mild to moderately atypical endothelium, whose nuclei projected into the vessel, consistent with a diagnosis of lowgrade AS. (Figure 6).

\section{DISCUSSION}

Classically, two risk factors are associated with skin and soft tissue AS: chronic lymphedema and radiotherapy. Exposures to carcinogens, such as thorium dioxide, arsenic and vinyl chloride, predispose to hepatic AS. About $3 \%$ of cases occur in the context of genetic
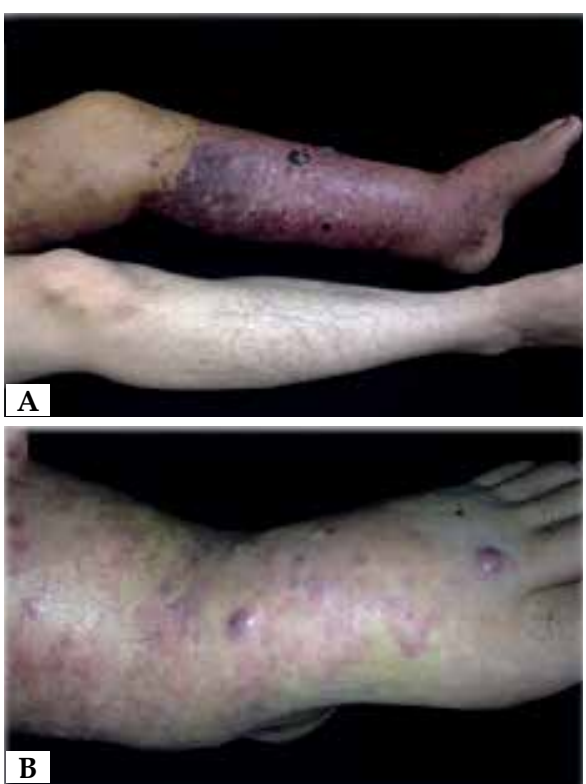

Figure 1: A. Red-to-purple confluent macules, papules and nodular lesions associated with chronic lymphedema on the left lower limb. B. Red-to-purple tumoral lesions

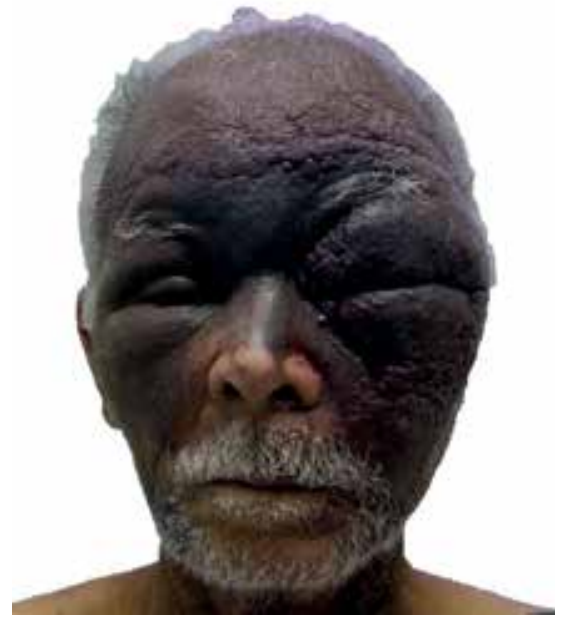

FIGURE 3: Extensive, infiltrative, confluent purple-black lesions on the scalp, frontal region and face, forming an easily bleeding left periorbital mass
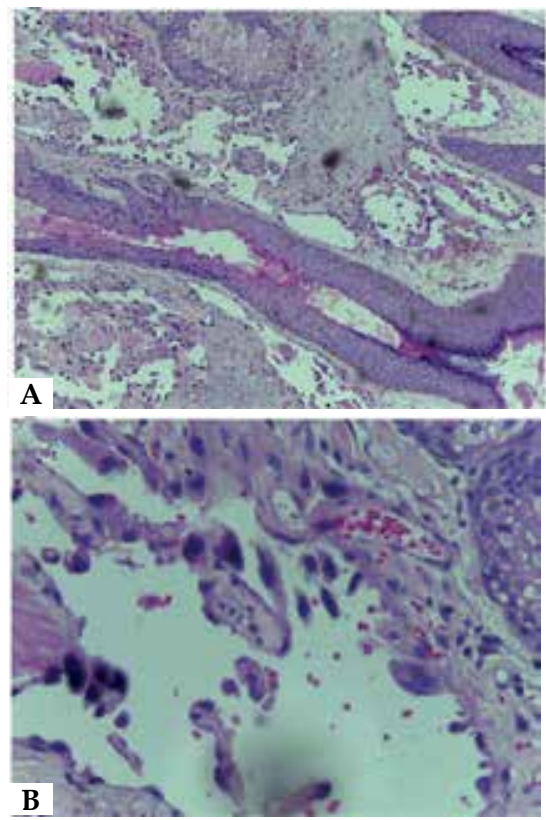

Figure 4: A. Proliferation of irregular, anastomosed clefts (HE, 100x). B. Atypical endothelium, with hyperchromatic nuclei, projecting into the lumen (HE, 400x).
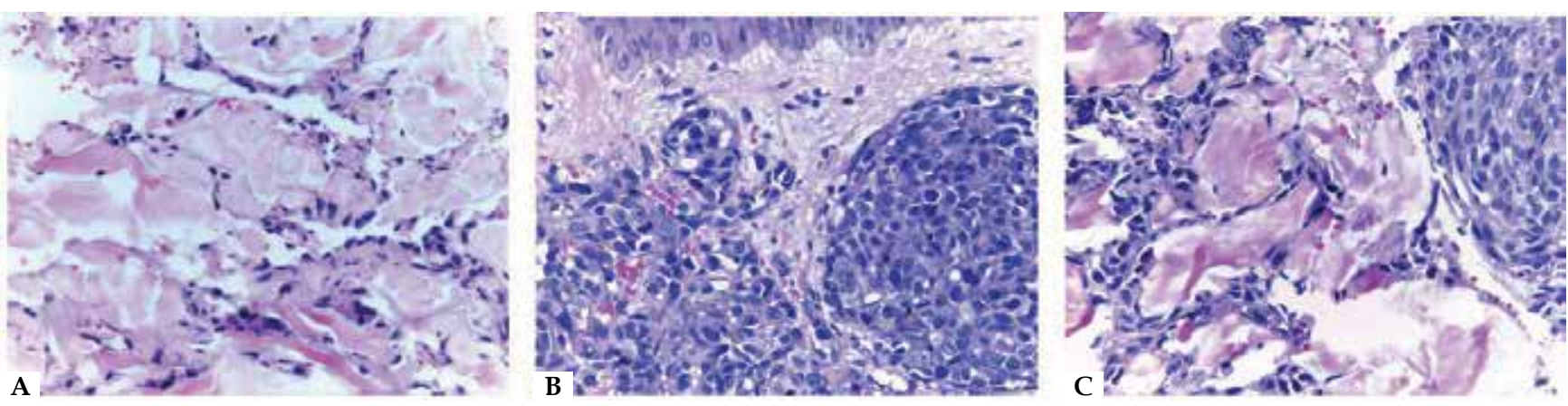

Figure 2: A. Irregular vascular clefts, consisting of endothelium with atypical and prominent nuclei towards the lumen (purple macule lesion) (HE, 100x). B. Epithelioid tumor masses, formed by atypical, large, rounded cells, with acidophilic cytoplasm and frequent mitotic figures (tumoral lesion) (HE, 100x). C. The two histological aspects visualized in the same field (HE, 100x) 
syndromes, mainly retinoblastoma and neurofibromatosis type $1 .^{1,2,4-7}$

The most common form of cutaneous AS is the one that occurs on the scalp and face of white men of advanced age (70 to 75 years), as in case 2. In this subtype, no etiological factor has proven participation. Clinically, typical lesions resemble bruises, are multifocal and infiltrative, and can ulcerate and bleed. ${ }^{2,3}$

Stewart-Treves syndrome, as observed in case 1 , is charac-
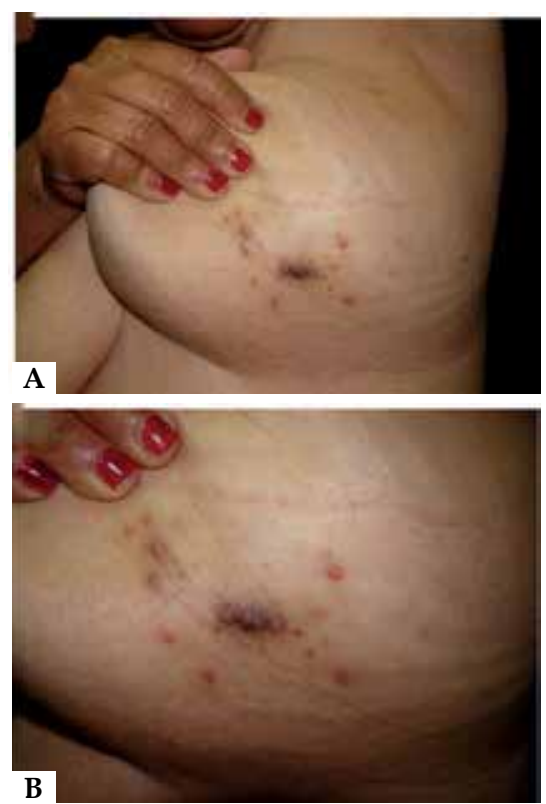

Figure 5: A. Papulotumoral, red-to-purple, hardened lesions in the lower lateral quadrant of the left breast. B. Central purple-black tumor lesion, surrounded by "satellites" reddish papules

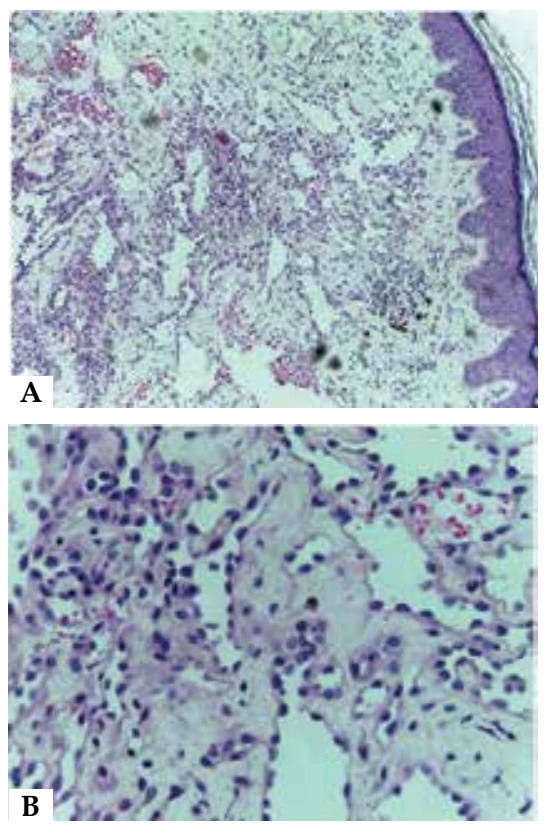

Figure 6: A. Anastomotic suture of sinusoidal vessels, with an infiltrative aspect $(\mathrm{HE}, 25 \mathrm{x})$. B. Endothelium with mild to moderate atypia and prominent nuclei into the vessel (HE, 100x) terized by the appearance of lymphangiosarcoma in a location affected by chronic lymphedema. The upper limb is the site involved in $90 \%$ of cases, usually in 5 to 15 years after radical mastectomy with axillary emptying for treatment of breast cancer. Several clinical lesions are described, such as palpable subcutaneous mass, difficult to heal ulcers with recurrent bleeding, bluish-red macules and nodules, among others. ${ }^{1-3,8,9} \mathrm{It}$ is believed that the accumulation of serum proteins in lymphedema induces immunosuppression and facilitation of vascular oncogenesis. ${ }^{8,9}$

AS associated with radiotherapy occurs mainly after treatment for breast cancer, and especially affects the dermis and subcutaneous tissue, often on the border of the irradiated area, but it is not exclusive to the breast. AS of the breast occurs in women of advanced age (67 to 71 years), with peak incidence 5 to 10 years after treatment, exhibiting a wide variety of clinical presentations, as in case 3.,2,5,10

Histological findings may vary both in a given case and among cases. The spectrum of findings includes irregular and anastomosed endothelial tissue in the most differentiated areas as well as epithelioid or spindle masses with a high degree of atypia, frequent mitoses, necrosis and hemorrhages in less differentiated areas. The pattern of dissecting proliferation between the collagen bundles and the nuclear projection towards the lumen are characteristic., ${ }^{1,6}$

AS typically expresses endothelial markers, such as CD34 and VEGF. CD31, von Willebrand factor and Ulex europaeus I lectin are more useful in less differentiated cases, being the most sensitive marker of hemangiosarcomas. Lymphatic vessel markers can also be expressed, such as podoplanin (D2-40). 2,4,6,8

Prognostic factors include age, extent of disease, primary site, tumor size and histological grade. ${ }^{2,3,7}$ Because of the difficulty of resection with free margins, tumors located in the head or neck present worse prognosis. ${ }^{3}$

AS outcome is bleak, and overall survival in five years is $35 \%$. Even with extensive resection, rates of relapse and early metastasis are high. The best chances of survival are observed in patients with localized disease that undergo aggressive surgery early. Radiotherapy and chemotherapy are palliative options for metastatic disease or adjuvants to surgical treatment. ${ }^{1,2,8}$ The three patients were transfered to the oncology reference hospital for staging and treatment. $]$ 


\section{REFERENCES}

1. Young RJ, Brown NJ, Reed MW, Hughes D, Woll PJ. Angiosarcoma. Lancet Oncol. 2010;11:983-91.

2. Penel N, Marréaud S, Robin YM, Hohenberger P. Angiosarcoma: State of the art and perspectives. Crit Rev Oncol Hematol. 2011;80:257-63.

3. Albores-Saavedra J, Schwartz AM, Henson DE, Kostun L, Hart A, AngelesAlbores D, et al. Cutaneous angiosarcoma. Analysis of 434 cases from the Surveillance, Epidemiology, and End Results Program, 1973-2007. Ann Diagn Pathol. 2011;15:93-7.

4. Wen VW, MacKenzie KL. Modeling humam endothelial cell transformation in vascular neoplasias. Dis Model Mech. 2013;6:1066-79.

5. Penel N, Grosjean J, Robin YM, Vanseymortier L, Clisant S, Adenis A. Frequency of certain established risk factors in soft tissue sarcomas in adults: A prospective descriptive study of 658 cases. Sarcoma. 2008;2008:459386.

6. Antonescu C. Malignant vascular tumors--an update. Mod Pathol. 2014;27:S30-8.

7. de Bree R, van der Waal I, de Bree E, Leemans CR. Management of adult soft tissue sarcomas of the head and neck. Oral Oncol. 2010;46:786-90.

8. Sharma A, Schwartz RA. Stewart-Treves syndrome: Pathogenesis and management. J Am Acad Dermatol. 2012;67:1342-8.

9. McHaffie DR, Kozak KR, Warner TF, Cho CS, Heiner JP, Attia S. Stewart-Treves syndrome of the lower extremity. J Clin Oncol. 2010;28:e351-2.

10. Arora TK, Terracina KP, Soong J, Idowu MO, Takabe K. Primary and secondary angiosarcoma of the breast. Gland Surg. 2014;3:28-34.

MAILING ADDRESS:

Aline Neves Freitas Cabral

Avenida Marechal Campos, 1.355

Santa Cecilia - Hospital Universitário Cassiano Antonio de Moraes - Serviço de Dermatologia Prof. Delio Delmaestro 29043-260 - Vitória - ES

Brazil

Email:alinenfcabral@gmail.com

How to cite this article: Cabral ANF, Rocha RH, Amaral ACV, Medeiros KB, Nogueira PSE, Diniz LM. Cutaneous angiosarcoma: report of three different and typical cases admitted in a unique dermatology clinic. An Bras Dermatol. 2017;92(2):235-8. 